\title{
In Vivo Antibacterial Activity of Dihydroanthracene Disulfonic Acid Derivative in a Case Diagnosed with Necrotizing Fasciitis
}

\author{
Florjana Rustemi, PhD Student \\ Albanian University, Albania \\ Ledjan Malaj, Prof. \\ Gezim Bocari, Prof. \\ University of Medicine, Tirana, Albania \\ Bledi Masati, PhD \\ University Hospital Center "Mother Teresa", Tirana, Albania
}

Doi: 10.19044/esj.2018.v14n30p28 URL:http://dx.doi.org/10.19044/esj.2018.v14n30p28

\begin{abstract}
This paper focuses on reporting the in vivo antibacterial activity of dihydroanthracene disulfonic acid derivative preparation in a case diagnosed with mixed flora infection with streptococci, enterobacteria, bacterium coli, pseudomonas and anaerobic proteus that resulted in a resistant to ampicillin, piperacillin, ceftazidim, imipenem, and which is moderately sensitive to cotrimoxazole and azithromycin. This resistant infection affected a patient with history of alcohol and tobacco consumption. It later became complicated with Necrotizing Fasciitis after colon cancer surgery. This study is an in vivo study, which is aimed to measure the antibacterial activity of our new preparation against multiresistant mixed bacterial infection. In the situation of this difficult and almost hopeless case, the patient started oral and local treatment with dihydroanthracene disulfonic acid derivative. Although the fatality of the case was due to the antibiotics resistance, the patient's treatment was attributed to the antibacterial effect of the preparation used. A significant improvement was observed in the first 24 hours after the beginning of the treatment. This happened when all parenteral antibiotics were interrupted. The improvement continued and within a couple of weeks, the wounds were clean and with visible granulation. After three months, the wounds cicatrized "per secundam". In order to have a general conclusion regarding the in vivo study related to the treatment with dihydroanthracene disulfonic acid derivative preparation, other results are needed. The significant effect shown in the above case, demonstrates that this preparation is promising for treating
\end{abstract}


life threatening resistant microorganisms infections. Further studies are needed to evaluate the full pharmacological activity of the new preparation.

Keywords: Antimicrobial activity, Necrotizing Fasciitis, dihydroanthracene disulfonic acid derivative preparation

\section{Introduction}

A male patient with the initials Z.P., 61 years old from Elbasan, diagnosed with Adenocarcinoma of the sigmoid colon (American Cancer Society, 2017), hospitalized in the University Hospital Center "Mother Theresa" Tirana, in the General Surgery Clinic underwent surgery on 19.11.2015 for "Anterior upper resection with temporary colostomy Hartmann's procedure" (Adams, Mann, Bokey, et al., 1992). The patient had a good postoperative decay, a functional colostomy on day three, without fever. On 25.11.2015, the patient left the hospital. He had a functional colostomy and diuresis about $2700 \mathrm{ml} / 24$ hours. Clinically, one week after the hospital dismissal, a wound infection appeared at the lower corner of the stoma of the patient. On 04.12.2015, the patient was present at the Emergency Department with a complicated infection of the wound, body temperature of $38.5^{\circ} \mathrm{C}$, complaining of severe pain on the left side of the back (Janeviscus, Hann, \& Butt, 1982) where red spots and crepitations during palpitation appeared. The patient was hospitalized and in addition to the surgery incision and the anus praeter, 24 parallel incisions in the median dorsal line were performed. The secretions smelled stingy because of the necrotic tissue present in the wounds. The patient was diagnosed with Necrotizing Fasciitis (Ahrenholz, 1988) complication post colon resection (Lancerotto, Tocco, Salmaso, et al., 2012; Rea, \& Wyrick, 1970; Wilson, 1952). Removal of the necrotic tissue and local treatment were performed twice daily and oxygenated water, potassium permanganate, and hypertonic solution were administered.

Antibiotics administered systemically comprises of:

1) Cefazoline three times daily $x 1.0 \mathrm{~g}$ intravenously;

2) Metronidazol three times daily $x 100 \mathrm{ml}$ intravenously; and

3) Gentamycine twice daily x $80 \mathrm{mg}$ intravenously.

The therapy employed also consists of:

4) Ranitidine four times daily x $50 \mathrm{mg}$ intravenously;

5) Dalteparine once daily x 5000 UI S.C injection;

6) $\mathrm{NaCl}$ solution $0.9 \%$ / $500 \mathrm{ml}, \mathrm{KCl}$ solution $7.5 \% / 20 \mathrm{ml}, \mathrm{CaCl}_{2} 10$ $\% / 5 \mathrm{ml} x$ three times daily intravenously;

7) Diclofenac twice daily x $75 \mathrm{mg}$ intravenously. 
On 12.12.2015, Cefazoline was replaced with Ceftazidime twice daily x $2.0 \mathrm{~g}$ intravenously. Despite the careful systemic and local treatment (Swain, Hatcher, Azadian, et al., 2013; Roemmele \& Batdorff, 2015), the measured body temperature was $38-39^{\circ} \mathrm{C}$ with a stingy odor from the wounds. The patient's situation was not improving. However, this indicates the gravity of the condition and the low effectiveness of the treatment. On 18.12.2015, all three antibiotics were removed and replaced with Moxifloxacine once daily x $400 \mathrm{ml}$ intravenously. Despite that, the situation did not improve and the toxicity increased. The clinical situation from 04.12.2015 to 17.12.2015 showed that despite the careful local treatment and the combination of antibiotics administered intravenously (Janeviscus, Hann, \& Butt, 1982), the treatment was not successful. Biochemical analysis determined anemia and impared hepatic function. Microbiological analysis resulted in mixed flora with streptococci, enterobacteria, bacterium coli, pseudomonas, and anaerobic proteus (Ahrenholz, 1988; Roemmele \& Batdorff, 2015; Vayvada, Demirdover, Menderes, et al., 2012). This, therefore, resulted in a resistant to ampicillin, amoxicillin, piperacillin, cephalotin, ceftazidim, gentamicin, imipenem, and is moderately sensitive to cotrimoxazole and azithromycin. After a multidisciplinary consultation with pharmacologists, evaluating the microorganism's resistance to antibiotics and impaired hepatic function, it was decided that the patient should stop taking the antibiotics. Clinically, the patient was in a critical condition which is getting worse every day. The Faculty of Medicine was engaged in the studies of the effects of dihydroanthracene disulfonic acid derivative.

\section{Methods}

\section{Study Design: Case Report}

This study is an in vivo study, which is aimed to measure the antibacterial activity of our new preparation against multiresistant mixed bacterial infection. In the situation of this difficult and almost hopeless case, following the patient's and family consent, it was decided that the patient should start an oral treatment with dihydroanthracene disulfonic acid derivative twice daily $\mathrm{x} 2 \mathrm{~g}$ and a local treatment with a $20 \%$ solution of dihydroanthracene disulfonic acid derivative. To evaluate the effectiveness of the in vivo treatment and the antibacterial activity of dihydroanthracene disulfonic acid derivative, on 17.12.2015, this preparation was applied locally only on one of the 24 wounds. It was very clear that after 24 hours, in the wound treated with the solution of the dihydroanthracene disulfonic acid derivative, the necrotic tissue was noticeably reduced and the margins of the treated wound became more vital. This was the evidence of the efficacy of the preparation used in vivo. 
Treatment: After this first step of testing the effectiveness and tolerance of the preparation, together with the local treatment as listed above, dihydroanthracene disulfonic acid derivative was used twice both locally and orally.

\section{Results}

From the first day of treatment, a decrease of the stingy odor up to $60 \%$ was observed. Only 24 hours after the first administration, the body temperature measured dropped to $37^{\circ} \mathrm{C}$ and the stingy odor of the wounds decreased noticeably. On the third to fifth day, cleaner wounds with visible granulation in the margins were observed. The measured body temperature was $37^{\circ} \mathrm{C}$. The stingy putrid odor decreased up to $80 \%$. Every day during the local treatment, necrotic tissue (Rea \& Wyrick, 1970; Giuliano, Lewis, Hadley, et al., 1977) as shown in Figure 1 were removed, but the amount of the necrotic tissue after the fourth day decreased.

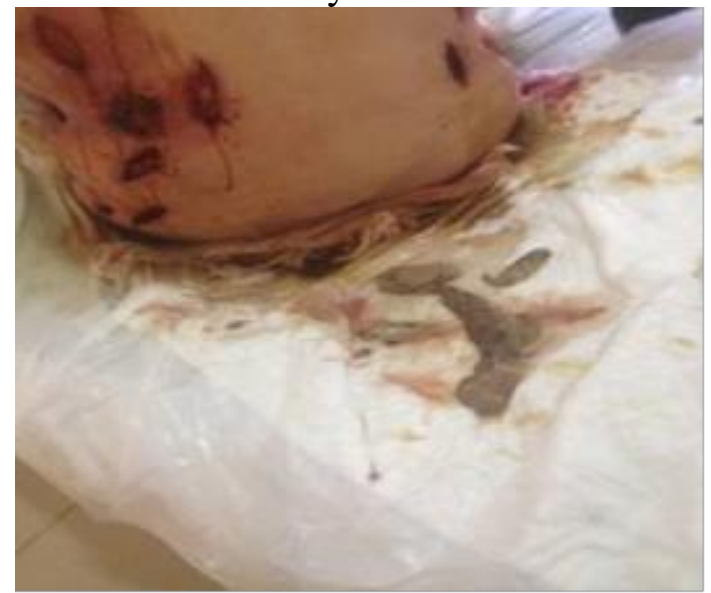

Figure 1. Necrotic tissue removed from the wounds

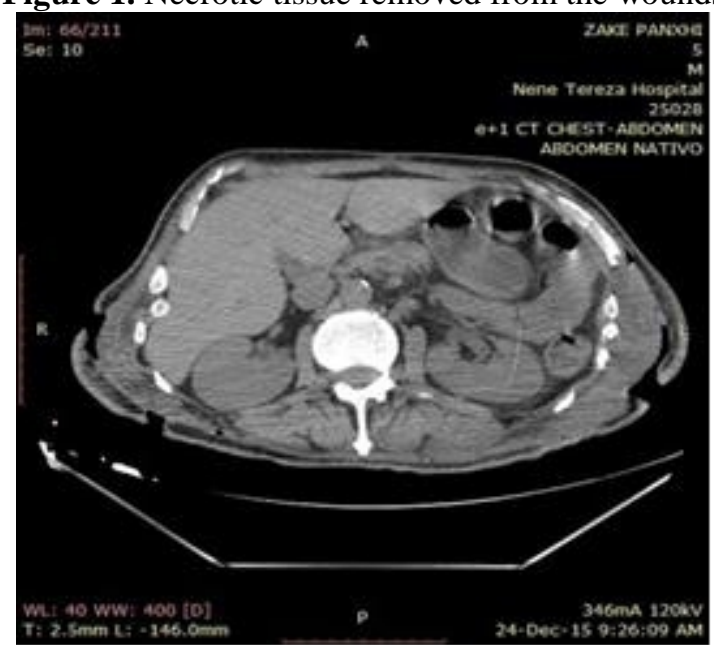

Figure 2. Scanner performed in patient 
The scanner image presented in Figure 2 performed on 24.12.2015 showed deep pockets in the wounds created after the mechanical removal of necrotic tissue $[6,11]$. On 28.12.2015, the patient was well nourished by mouth, slept well and was afebrile. On 08.01.2016, a clean wounded patient without necrotic tissue (Rea \& Wyrick, 1970; Giuliano, Lewis, Hadley, et al., 1977) and pus was transferred to his birthplace in Elbasan hospital. During the treatment of this fatal case, the condition of the patient improved until recovery. Thus, the situation of the patient improved as shown below in Figures 3-11. When all parenteral antibiotics were stopped, the local treatment of one of the 24 wounds after 24 hours showed clear improvement. The next day following oral treatment and local treatment, the body temperature dropped to $37^{\circ} \mathrm{C}$. The 24 incisions with purulent secretions cleared quickly. The results confirmed the in vivo antibacterial activity of the preparation against mixed bacterial flora with resistant life threatening microorganisms (Ahrenholz, 1988). With this new treatment, the infection was dominated within three to four days and the bad odor of the wounds almost disappeared indicating a fast dominance of anaerobes. After five to six days of treatment, the granulation of the wounds began. The total area of 24 wounds from about $200 \mathrm{~cm}^{2}$ reduced significantly and after two months all the wounds were closed cicatrising "per secundam".

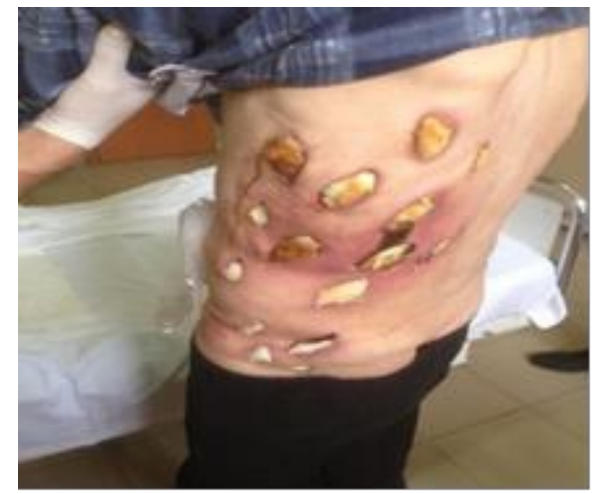

Figure 3. Wounds conditions on the left part of the body on 04.01.2016

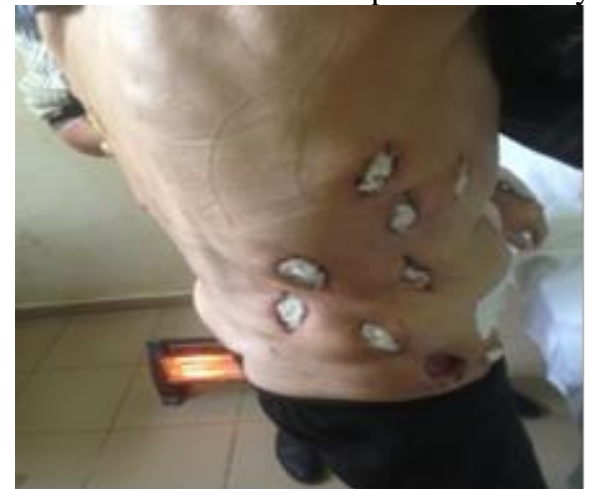

Figure 4. Wounds conditions on the right part of the body on 04.01.2016 


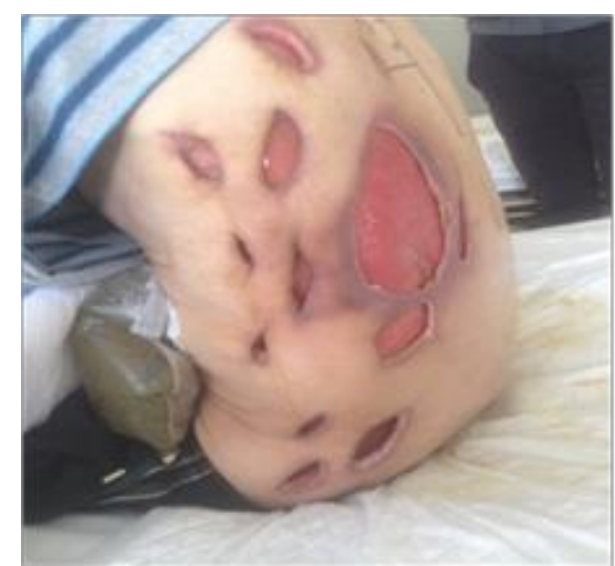

Figure 5. Wounds conditions on 16.01.2016

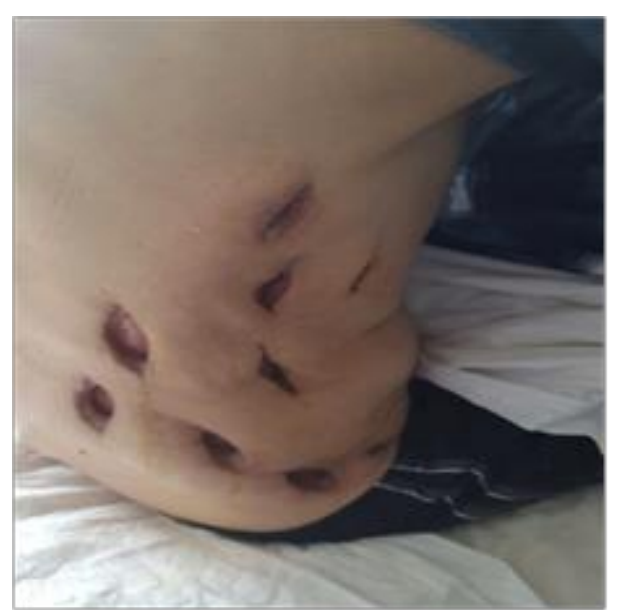

Figure 6. Wounds conditions on the left part of the body on 23.01.2016

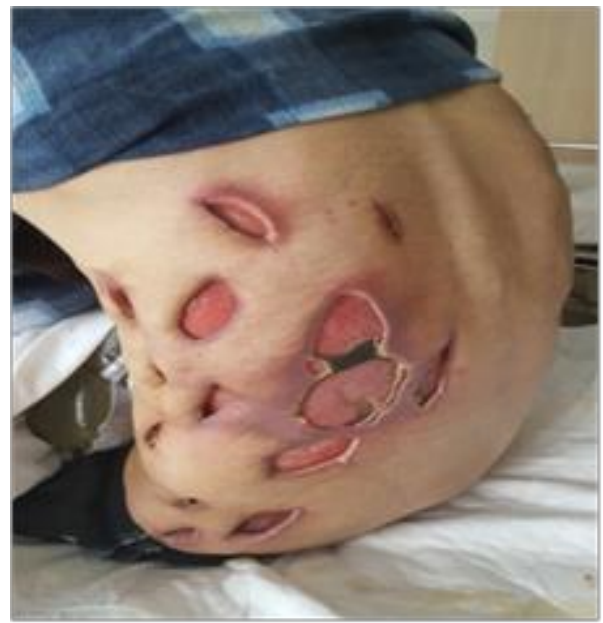

Figure 7. Wounds conditions on the right part of the body on 23.01.2016 


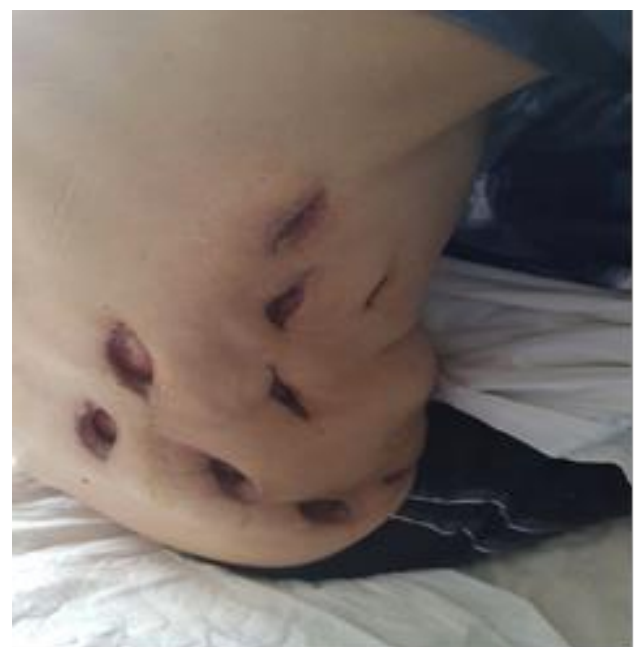

Figure 8. Wounds conditions on 12.02.2016

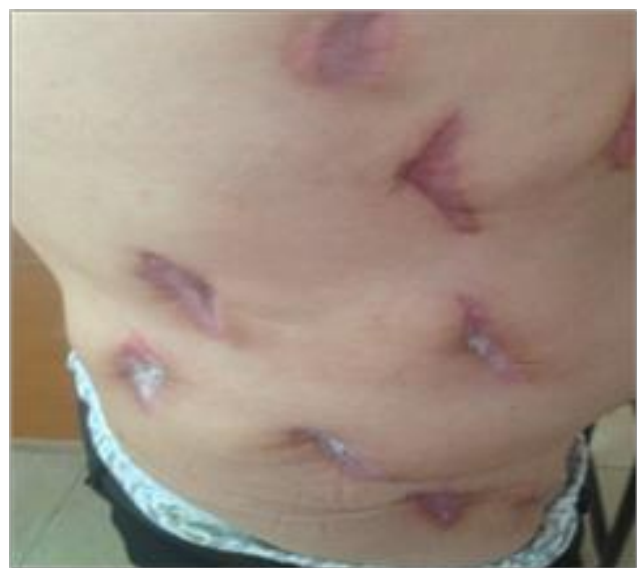

Figure 9. Wounds conditions on 03.03.2016

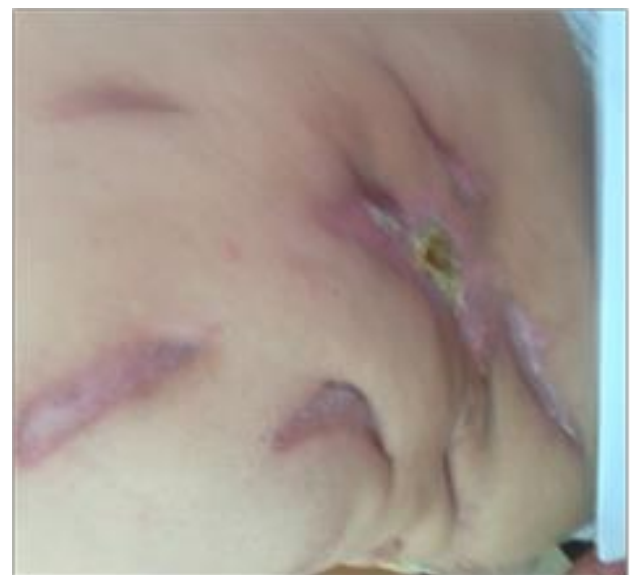

Figure 10. Wounds conditions on 21.03.2016 


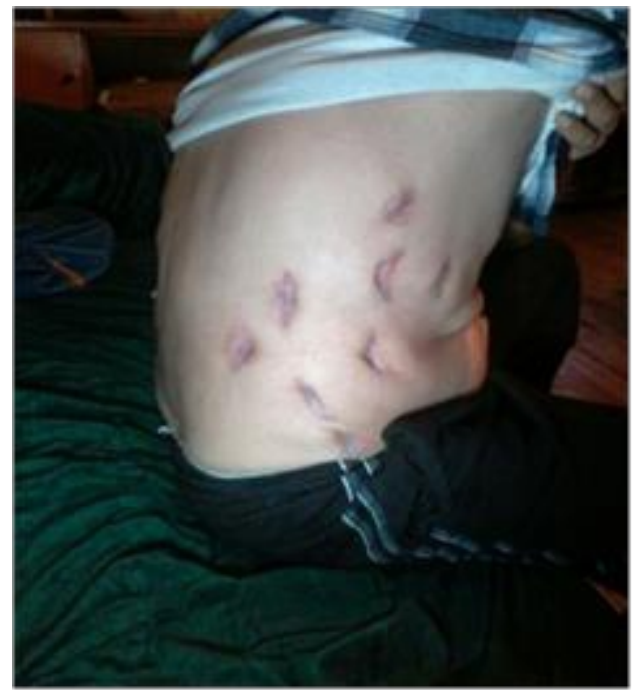

Figure 11. Wounds conditions on 29.04.2016

\section{Discussion}

Necrotizing Fasciitis is a severe, rare, potentially lethal soft tissue infection that develops in the scrotum and perineum, the abdominal wall, or the extremities. The infection progresses rapidly, and septic shock may ensue; hence, the mortality rate is high (median mortality $32.2 \%$ ) that approaches $100 \%$ without treatment (Misiakos, Bagias, Patapis, et al., 2014). Necrotizing fasciitis is known throughout the world for its high lethality (Ahrenholz, 1988; Swain, Hatcher, Azadian, et al., 2013; Roemmele \& Batdorff, 2015). Nevertheless, this case was fatal because the resistance to all antibiotics and curing of the patient is attributed to the antibacterial effectiveness of the used preparation dihydroanthracene disulfonic acid derivative due to the fact that significant improvement was observed in the first 24 hours after the beggining of the treatment, especially after all parenteral antibiotics were stopped. The improvement progressed and within weeks, the wounds were clean and with visible granulation. Undoubtedly, the in vivo results of dihydroanthracene disulfonic acid derivative are very promising, and they may be adopted by more physicians in the future.

\section{Conclusion}

In order to have a general conclusion regarding the in vivo study related to the treatment with dihydroanthracene disulfonic acid derivative, other results are needed. Thus, the evidence shown in the above case demonstrates that this preparation had an antibacterial activity on such a serious and critical life threatening situation which allows us to confirm that it will act equally in resistant microorganisms solitary wounds. 


\section{References:}

1. Adams, WJI., Mann, LJ., Bokey, EL., Chapuis, PH., Koorey, SG., \& Hughes, WJ. (1992). Hartmann's procedure for carcinoma of the rectum and sigmoid colon. The Australian and New Zealand journal of surgery. Mar;62(3):200-3. Available from: https://www.ncbi.nlm.nih.gov/pubmed/1372498

2. Ahrenholz, DH. (1988). Necrotising soft-tissue infections. Surg Clin North Am.;68:199-214. [PubMed]

3. American Cancer Society (2017). Understanding Your Pathology Report: Invasive Adenocarcinoma of the Colon. February. Available from: https://www.cancer.org/treatment/understanding-yourdiagnosis/tests/understanding-your-pathology-report/colonpathology/invasive-adenocarcinoma-of-the-colon.html

4. Evangelos, P., Misiakos, GB., Paul, P., Dimitrios, S., Prodromos, K., \& Anastasios, M. (2014) Current Concepts in the Management of Necrotizing Fasciitis. Front Surg.; 1:36. Published online 2014 Sep 29. Prepublished online, May 31. doi:10.3389/fsurg.2014.00036

5. Giuliano, A., Lewis, F.Jr., Hadley, K., \& Blaisdell, FW. (1977). Bacteriology of necrotising faciitis. Am J Surg.; 134:52-7. [PubMed].

6. Jacqueline, A. \& Roemmele, DB. (2015). Surviving the Flesh-Eating Bacteria Understanding, Preventing, Treating, and Living with the Aftermath of Necrotising Fasciitis; Second Edition; July; 84-108

7. Janeviscus, RV., Hann, S., \& Butt, MD. (1982). Necrotising fasciitis. Surg Gynecol Obstet.; 154:97-102. [PubMed].

8. Lancerotto, L., Tocco, I., Salmaso, R., Vindigni, V., Bassetto, F. (2012). Necrotising fasciitis: Classification, diagnosis, and management. J Trauma Acute Care Surg.; 72:560-6. [PubMed].

9. Rea, WJ. \& Wyrick, WJ. (1970). Necrotising fasciitis. Ann Surg.; 172:957-64. [PMC free article] [PubMed].

10. Swain, RA., Hatcher, JC., Azadian, BS., et al. (2013). A five-year review of necrotising fasciitis in a tertiary referral unit. Annals of The Royal College of Surgeons of England Vol. 95(1):57-60, January.

11. Vayvada, H., Demirdover, C., Menderes, A., \& Karaca, C. (2012). Necrotising fasciitis: diagnosis, treatment and review of the literature. Turkish Journal of Trauma \& Emergency Surgery (Ulus Travma Acil Cerrahi Derg.) November; 18(6):507-13.

12. Wilson, B. (1952). Necrotising fasciitis. Am Surg.;18:416-31. [PubMed]. 